Draft Version March 21, 2022

Preprint typeset using $\mathrm{LAT}_{\mathrm{E} X}$ style emulateapj v. 6/22/04

\title{
ON USING THE CMD MORPHOLOGY OF M67 TO TEST SOLAR ABUNDANCES
}

\author{
Z. Magic And A. Serenelli ${ }^{1}$ And A. Weiss \\ Max-Planck-Institut für Astrophysik, Karl-Schwarzschild-Str. 1, 85748 Garching, Germany \\ AND \\ B. Chaboyer \\ Dartmouth College, Hanover, NH, USA \\ Draft version March 21, 2022
}

\begin{abstract}
The open cluster M67 has solar metallicity and an age of about 4 Gyr. The turn-off mass is close to the minimum mass for which solar metallicity stars develop a convective core during main sequence evolution as a result of the development of hydrogen burning through the CNO-cycle. The morphology of the color-magnitude-diagram (CMD) of M67 around the turn-off shows a clear hook-like feature, direct sign that stars close to the turn-off have convective cores. VandenBerg et al. investigated the possibility of using the morphology of the M67 turn-off to put constraints on the solar metallicity, particularly CNO elements, for which solar abundances have been revised downwards by more than $30 \%$ over the last few years. Here, we extend their work filling in the gaps in their analysis. To this aim, we compute isochrones appropriate for M67 using new (low metallicity) and old (high metallicity) solar abundances and study whether the characteristic turn-off in the CMD of M67 can be reproduced or not. We also study the importance of other constitutive physics on determining the presence of such a hook, particularly element diffusion, overshooting and nuclear reaction rates. We find that using the new solar abundance determinations, with low CNO abundances, makes it more difficult to reproduce the characteristic CMD of M67. This result is in agreement with results by VandenBerg et al. However, changes in the constitutive physics of the models, particularly overshooting, can influence and alter this result to the extent that isochrones constructed with models using low CNO solar abundances can also reproduce the turn-off morphology in M67. We conclude that only if all factors affecting the turn-off morphology are completely under control (and this is not the case), M67 could be used to put constraints on solar abundances.
\end{abstract}

Subject headings: Sun: abundances — open clusters and associations: individual (M67)

\section{INTRODUCTION}

The latest revision of the solar abundances by Asplund et al. (2005, AGS05) resulted in a drastic reduction of the abundances of carbon, nitrogen, and oxygen, and thus in the total metallicity of the Sun. While $Z / X$ in the previous standard references by Grevesse \& Noels (1993, GN93) and Grevesse \& Sauval (1998, GS98) was 0.0245 respectively 0.0230 , Asplund et al. (2005) determined a value of 0.0165 . The recently published complete re-analysis of all elements by Asplund et al. (2009, AGS09) corrected this number slightly upwards to 0.0183 . It is interesting to realize that $Z / X$ has constantly gone down with time since Anders \& Grevesse (1989), who gave a value of 0.0275 .

While the analysis by Asplund et al. (2005) and in particular by Asplund et al. (2009) is undoubtedly the most complete, coherent and technically advanced one, the results are far from being accepted. The reason is that standard solar models (SSM), calculated for the new metal abundances, agree much less with well established and accurately determined results from helioseismology: the sound speed and density profile, the depth of the convective envelope, and its helium content. Bahcall et al. (2005a) investigated the new "solar model problem", which has been confirmed in many sub-

\footnotetext{
Electronic address: magic@mpa-garching.mpg.de

${ }^{1}$ Instituto de Ciencias del Espacio (CSIC), Facultad de Ciències, Campus UAB, 08193, Bellaterra, Spain
}

sequent, independent publications. Antia \& Basu (2006) further demonstrated that helioseismic data require the solar envelope to have a composition close to the old photospheric abundances, and Chaplin et al. (2007) used low-degree p-modes penetrating to the solar core to arrive at a similar conclusion.

To cure the "trouble in paradise" (Asplund et al. 2005) attempts were undertaken to confirm the previous, higher abundances of intermediate elements, in particular of oxygen (e.g. Centeno \& Socas-Navarro 2008; Caffau et al. 2008). On the other hand, the lower abundances were recovered, too (Maiorca et al. 2009 for nitrogen; Socas-Navarro \& Norton 2007 for oxygen).

On the side of the solar model community, possible or necessary changes to the constitutional physics were discussed to restore the previous excellent agreement with helioseismology (see Guzik 2008 for a summary). In particular, an increase of opacities has been considered the most promising approach (Basu \& Antia 2004; Bahcall et al. 2005b; Antia \& Basu 2006; Christensen-Dalsgaard et al. 2009). But updated Rosseland opacities for the solar interior are not enough to solve the solar abundance problem (Bahcall et al. 2005c). Also, a postulated upward correction to the neon abundance has not found observational support (Young 2005).

It is therefore reasonable to shift attention also to other stellar objects, since any revision of the solar metallicity yardstick leads to a corresponding change in the metal- 
licity of all stars. This is in particular true for stars with $[\mathrm{Fe} / \mathrm{H}] \approx 0$, since their metallicity is often determined in a strictly differential and therefore quite accurate way. Alecian et al. (2007) found that with the lower AGS05 solar composition they could reproduce the properties of the pre-main sequence binary system RS Cha, while it was impossible to fulfill all observational constraints with the higher GN93 abundances.

Another obvious test was performed by VandenBerg et al. (2007, VG07), using the CMD morphology of the open cluster M67, which has a metallicity of $[\mathrm{Fe} / \mathrm{H}]=0.00 \pm 0.03$ (Randich et al. 2006) and a turn-off (TO) age around 4 Gyr (e.g. Pietrinferni et al. 2004). At this age the turn-off mass is slightly higher than $1 M_{\odot}$ and close to the critical mass at which a convective core on the main-sequence appears due to the dominance of the CNO-cycle over the pp-chain (Kippenhahn \& Weigert 1990). The CMD of M67 (Montgomery et al. 1993; Sandquist 2004) shows a clear hook-like structure at the turn-off, indicative of a convective core for the turn-off stars. Since the efficiency of the CNO-cycle is directly proportional to the abundance of these elements, the reduced solar abundances of AGS05 could possibly result in a radiative core for stars at the TO, although in the fitting process the change in luminosity (at given mass, luminosity is lower for the AGS05 mixture) and effective temperature complicates simple predictions about the TO-mass and core structure. Indeed, VG07 found that the TO-hook disappeared for the new abundances, thereby strengthening the case for the GS98 metallicity scale.

However, VG07 did not fail to mention some caveats: Their models were calculated without atomic diffusion taken into account, a physical process that at least for solar models - irrespective of the metallicity scale used - is essential for the best possible agreement with seismic inferences. They correctly pointed out that diffusion helps to support a convective core at a stellar mass lower than in models ignoring this effect (Michaud et al. 2004), mainly due to the increase of CNO abundances in the stellar core by gravitational settling. The different ages of M67 and the Sun, but identical present-day abundances could also imply different initial abundances, an effect that should be taken into account in careful and precise studies. Apart from these points, one should keep in mind that with the occurrence of a convective core the question about the amount of overshooting arises. Its treatment, and in particular the value of any free parameter in its practical implementation, cannot simply be taken for granted, as usually such "calibration" was based on stellar models resting on the higher solar metallicity scale. Finally, the occurrence of a convective core may also depend on other aspects and therefore any conclusion concerning the metallicity is valid only under the assumption that all other influences are under control.

Given the result by VG07 and the open questions raised above, we revisited $\mathrm{M} 67$, trying to complete and extend the pioneering study of VG07. In $\S 2$ we will introduce briefly the stellar evolution code we have used for most of the calculations in the present work, and those aspects of the input physics, besides the solar metallicity, we will also investigate. In $\S[3$ we demonstrate that we can recover the results of VG07, a fact that is not irrelevant in the light of the following section. We then present our preferred model, showing that in this case the CMD morphology of M67 can be reproduced for both solar abundance scales. In $\S 4$ we investigate, how other approaches and variations of the input physics may also influence the quality of isochrone fits, and also possible systematic differences arising from the use of different stellar evolution codes. Our conclusions will follow in $\S[5$.

\section{STELLAR MODELS AND CRUCIAL DATA}

\subsection{GARSTEC}

Most of the stellar model calculations presented in this paper were done with the GARSTEC Weiss \& Schlattl 2008) program. Here, we briefly present aspects or modifications of the code, which are relevant for this work. The standard assumptions and settings can be found in the quoted reference.

Convective overshooting is treated as a diffusive process according to the approach described by Frevtag et al. (1996). The exact implementation can be found in Herwig et al. (1997). Based on hydrodynamical simulations, the diffusive constant therein $\left(D_{\mathrm{os}}\right)$ is assumed to decay exponentially beyond the formal Schwarzschild-border as

$$
D_{\mathrm{os}}(z)=D_{0} \exp \frac{-2 z}{f H_{P}}, D_{0}=\frac{1}{3} v_{0} \cdot H_{P} .
$$

This is equivalent to exponentially declining velocities of convective elements. In (1) $z$ is the radial distance from the Schwarzschild border and $H_{P}$ the pressure scale height taken there. The constant $D_{0}$ sets the scale of diffusive speed, and depends on the convective velocity $v_{0}$ inside of the convective border. $f$ is a free parameter defining the scale of the overshooting. It is known that fitting the CMD of young open clusters usually leads to an overshooting region extending for about $0.2 H_{P}$ in the classical local prescription. We have computed some test models for stars between 2 and $6 M_{\odot}$ and found that this overshooting value is well reproduced by the diffusive approach, during the hydrogen core burning phase, with a value of about $f=0.018$, a confirmation of previous results by Herwig et al. (1997).

For small convective cores, the amount of overshooting has to be limited. In the local description this is done, for example, by a multiplicative factor, which increases linearly with mass in the range of 1 to $2 M_{\odot}$ (see Pietrinferni et al. 2004, for an example). In our code this is achieved by a geometrical cutoff, where $H_{P}$ in Equation 1 is replaced by

$$
\widetilde{H_{P}}:=H_{P} \cdot \min \left[1,\left(\frac{\Delta R_{\mathrm{cz}}}{H_{P}}\right)^{2}\right],
$$

where $\Delta R_{\mathrm{cz}}$ is the thickness of the convective zone. In this way, the geometric cutoff has the advantage that the overshooting region is always limited to a fraction of the extension of the convective region. It is our standard limiting procedure for overshooting. A similar cut is used in Ventura et al. (2005, 1998).

An alternative approach, namely the use of a ramp function for the $f$ parameter, is also applied for some of our calculations. For masses lower or equal than $1.1 M_{\odot}$ we use $f=0$ (i.e. no overshooting), whereas for masses equal to or higher than $2.0 M_{\odot}$ we set $f=0.018$. In the 
intermediate mass range the efficiency of overshooting varies linearly with mass

$$
f=\left(0.13 M / M_{\odot}-0.098\right) / 9 .
$$

The small discontinuity in the overshooting efficiency is of no consequence for our study. $1.1 M_{\odot}$ models with $f=0.005$, as would result from application of the above equation to this mass, lead, given the low overshooting efficiency, to the same evolutionary tracks as models without overshooting. This prescription for the overshooting efficiency leads to evolutionary tracks that reproduce well those by Pietrinferni et al. (2004).

Atomic diffusion is treated within the same diffusive numerical scheme. In this paper, hydrogen, helium and heavier elements (including iron) are diffusing. Radiative levitation is not taken into account.

To agree with the physical assumptions in VG07, the default set of nuclear reaction rates are those of the NACRE collaboration (Angulo et al. 1999). To investigate their influence, for individual reactions alternative rates were used. For the ${ }^{14} \mathrm{~N}(p, \gamma)^{15} \mathrm{O}$ reaction, we also used the rate from Marta et al. (2008), the newest result from the LUNA collaboration, which is lower by about a factor of two with respect to the NACRE rate at the relevant temperatures. This has a considerable effect on the TO morphology. Another reaction that turned out to be of surprisingly strong influence is the ${ }^{17} \mathrm{O}(p, \alpha)^{14} \mathrm{~N}$ reaction. We tested this by employing either the recent measurements by Moazen et al. (2007), which for $T>4 \times 10^{8}$ $\mathrm{K}$ is very similar to the NACRE recommendation, or the one given by Caughlan \& Fowler (1988). A detailed discussion on the reaction rates can be found in $\S 4.2$.

For all solar composition choices (AGS05, GS98) consistent Rosseland mean opacity tables were prepared following the procedure described in Weiss \& Schlattl (2008).

The final step to compare isochrones with observed CMDs is the transformation to colors. For this we used that by VandenBerg \& Clem (2003, VC03) which, together with a choice for the distance modulus and reddening of M67, results in satisfying CMD fits on the main sequence and subgiant branch. Alternatively, we used the transformations by Cassisi et al. (2004) for testing purposes.

The initial stellar parameters $\left(Y_{\text {in }}, Z_{\text {in }}\right.$, mixing length parameter) are obtained from solar model calibrations. This will be discussed in more detail in $\S 3.2$ With these, we computed the evolution from the zero-age mainsequence (ZAMS) to the tip of the Red Giant Branch (RGB) for mass values from 0.6 to $1.5 M_{\odot}$ in steps of $0.1 M_{\odot}$. We recall here that, as in VG07, we are not interested in considering the RGB of M67, where deficiencies in our treatment of the outer layers of the star could have an impact on the location of the models in the HRD. We confirmed by tests starting from the pre-main sequence that for stars with mass below the critical mass for the occurrence of a convective core, $M_{\text {ccc }}$, the transient convective core at the end of the pre-main sequence phase also appears here. This convective core is the result of the short phase of $\mathrm{CN}$ conversion, but may be sustained if convective overshooting is included. Finally, for constructing isochrones, the tracks are normalized to the so-called equivalent points (Bergbusch \& Vandenberg 1992; Pietrinferni et al. 2004) and the interpolation to an isochrone is done between the normalized tracks. After an isochrone age was fixed, we calculated an additional model with the TO-mass and recalculated the isochrone to make sure that the TOmorphology does not depend on the isochrone interpolation scheme.

\subsection{Data for $M 67$}

To compare our models with M67 we used the photometric data by Sandquist (2004). These are more accurate than the older data from Montgomery et al. (1993), which were used by VG07, and contain bona fide single stars only. Thus, this CMD has a narrower mainsequence band. However, we also tested some of our isochrones with the data by Montgomery et al. (1993). We used both $(B-V)$ and $(V-I)$ colors.

The dereddened distance modulus to M67 is $(m-$ $M)_{V}=9.70$ according to VG07, and $9.72 \pm 0.05$ (Sandquist 2004) based on subdwarf fitting to the lower main-sequence following Percival et al. (2003). The reddening is $E(B-V)=0.038$ (VG07) in good agreement with Sarajedini et al. (1999), who gave 0.04 \pm 0.02 . Similar values have been recently obtained by Twarog et al. (2009). For the metallicity we assumed a value of $[\mathrm{Fe} / \mathrm{H}]=0.0$, which is well within spectroscopically determined errors, for example by Gratton (2000), who gave $[\mathrm{Fe} / \mathrm{H}]=0.02 \pm 0.06$.

\subsection{Model composition}

To be consistent with VG07, we used for the old respectively new solar abundances those by GS98 and AGS05, although the former are simply an update of GN93 taking into account additional literature values, and AGS09 would be the most recent and complete re-analysis. Since AGS09 abundances are slightly higher than AGS05, our choice is testing the more extreme case. The initial abundances of the stellar models for the M67 isochrones are taken to be the same as those resulting from solar model calibrations.

\section{STANDARD FITTING PROCEDURES}

\subsection{Recovering VG07}

The idea of VG07 to use M67 for testing the effect of the new solar abundances rests on the fact that the turnoff mass of this open cluster is very close to the critical mass for the onset of core convection, $M_{\mathrm{ccc}}$, or "transition mass" (VandenBerg et al. 2007). This transition mass depends on the average exponent of the energy generation rate in the core (Kippenhahn \& Weigert 1990, Chap. 22), that increases with the contribution from the CNO-cycle, for which $\epsilon_{\mathrm{CNO}} \sim T^{15}$, in contrast to $\epsilon_{\mathrm{pp}} \sim T^{5}$ for the pp-chains at the relevant temperatures. The importance of the CNO-cycle depends on the nuclear reaction rates (see $\S 4.2$ ) as well as on the amount of "catalysts", i.e. the sum of the CNO-abundances. These are, incidentally, the elements with the largest reduction in their abundance according to AGS05. Therefore, the morphological change in the CMD, i.e. displaying the characteristic hook at the TO, or a gap in star density, can be used to determine whether the TO-mass is below or above $M_{\text {ccc }}$.

However, the sensitivity of the M67 TO morphology implies that also other effects may influence it, apart 


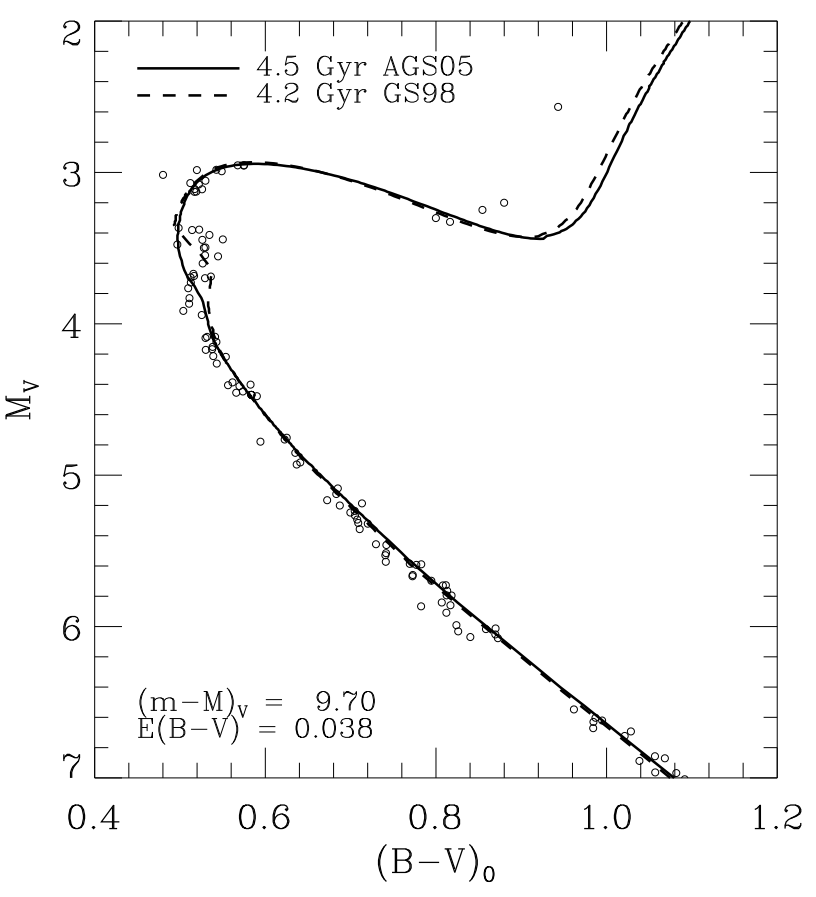

FIG. 1.- Isochrone fit to M67 for the two solar mixtures GS98 and AGS05 using a solar model calibration as in VG07 (see Table1) and NACRE nuclear reaction rates. Isochrone ages are indicated; distance modulus and reddening agree with VG07

from the metallicity. The nuclear reaction rates were already mentioned. Other possible aspects could be the amount of overshooting, atomic diffusion, and the premain sequence history. Last, but not least, technical details of the stellar evolution codes may play a role. It is therefore necessary to show that our results do not depend on the particular numerical code. As a first, and crucial step, we have successfully attempted to reproduce the key result by VG07. This will also show where changes to their procedure are indicated.

The first step concerns the solar model calibration. Here, as for the M67 models, VG07 used NACRE nuclear reaction rates, the OPAL (Iglesias \& Rogers 1996) and Ferguson et al. (2005) opacities (as is done in our code), but ignored diffusion. This point is crucial, as we will show later on. Some amount of convective overshooting (see $\S$ 4.1) was included for the M67 models, but is not relevant for the solar calibration. For the atmospheres they used the MARCS model (Gustafsson 2003). In this respect we differ, since we use standard Eddington grey atmospheres. However, as shown by VandenBerg et al. (2008), this has in our present case no significant influence on the tracks on the main-sequence and subgiant branch.

Table 1 contains in the first two rows the resulting solar model parameters by VG07 and in rows 3 and 4 the equivalent ones obtained with our code. Additionally, we also show the results when using the Dartmouth and LPCODE codes $(\S 4.3)$. Note that the mixing length parameters can never be compared in their absolute values due to the different formulations of MLT. Overall, the agreement with VG07 for the initial abundances is
TABLE 1

RESUlTS OF SOLAR CALIBRATIONS FOR DIFFERENT CODES AND SOLAR ABUNDANCES (COLS. 1 AND 2) AND FOR CASES WITH AND Without DifFUSION (COL. 3). $Y_{\text {in }}$ AND $Z_{\text {in }}$ ARE THE INITIAL HELIUM AND METAL ABUNDANCES. $\Delta$ DENOTES THE RELATIVE DIFFERENCE WITH RESPECT TO VG07 FOR THE CORRESPONDING ABUNDANCE.

\begin{tabular}{cccccccc} 
Code & Mixture Diffusion & $\alpha_{\mathrm{MLT}}$ & $Y_{\text {in }}$ & $\Delta$ & $Z_{\text {in }}$ & $\Delta$ \\
\hline \hline VG07 & GS98 & no & 1.84 & 0.26760 & & 0.01650 & \\
& AGS05 & no & 1.80 & 0.25590 & & 0.01250 & \\
\hline GARSTEC & GS98 & no & 1.59 & 0.26109 & $-2 \%$ & 0.01661 & $1 \%$ \\
& AGS05 & no & 1.62 & 0.25018 & $-2 \%$ & 0.01217 & $-3 \%$ \\
& GS98 & yes & 1.72 & 0.26903 & $1 \%$ & 0.01866 & $13 \%$ \\
& AGS05 & yes & 1.75 & 0.25939 & $1 \%$ & 0.01380 & $10 \%$ \\
\hline Dartmouth & GS98 & no & 1.78 & 0.26461 & $-1 \%$ & 0.01659 & $1 \%$ \\
& AGS05 & no & 1.77 & 0.24991 & $-2 \%$ & 0.01219 & $-3 \%$ \\
& GS98 & yes & 1.94 & 0.27419 & $2 \%$ & 0.01889 & $14 \%$ \\
& AGS05 & yes & 1.91 & 0.26075 & $2 \%$ & 0.01405 & $12 \%$ \\
\hline LP & GS98 & no & 1.71 & 0.26466 & $-1 \%$ & 0.01648 & $-0.1 \%$ \\
& AGS05 & no & 1.66 & 0.25026 & $-2 \%$ & 0.01217 & $-3 \%$ \\
& GS98 & yes & 1.85 & 0.27053 & $-1 \%$ & 0.01806 & $10 \%$ \\
& AGS05 & yes & 1.79 & 0.25728 & $1 \%$ & 0.01340 & $7 \%$
\end{tabular}

within $1-2 \%$, with our codes returning systematically lower initial metallicities (by 3\%) for the AGS05 mixture. Although a small effect, it additionally disfavors the appearance of a convective core in the M67 TO.

Using these initial composition values we calculated stellar tracks and isochrones for M67, which we show in Figure 1. The numerical values for distance and reddening are identical to VG07. The best-fitting isochrones have somewhat higher ages (by $0.3 \mathrm{Gyr}$ ). The TO mass is 1.229 respectively $1.200 M_{\odot}$ for the old and new composition. One recognizes the same basic result as in VG07: for the AGS05 mixture, the isochrone does not show the characteristic hook. However, it displays a slight inclination indicating the presence of a very small convective core at the TO mass. Consistent with this morphological sign, the TO mass is marginally higher than $M_{\mathrm{ccc}}$, which is $1.175 M_{\odot}$, while in the case of the GS98 mixture it is clearly above $M_{\mathrm{ccc}}=1.139 M_{\odot}$. These values appear to be very similar to those found by VG07 (see their Table 1). This exercise demonstrates that we are able to reproduce correctly VG07 and that their result is independent of the stellar evolution code used.

\subsection{Inclusion of diffusion}

VG07 already emphasized that atomic diffusion (effectively sedimentation) could modify this result as it leads to an increase of metallicity in the core over time. As explained in the previous subsection, this will favor the occurrence of a convective core and reduce $M_{\text {ccc }}$. However, the solar model calibration should also include diffusion, as it was shown that only with this physical effect the best agreement with the seismic Sun can be achieved. Only in this case the solar parameters, in particular the initial composition is determined as accurately as possible.

The corresponding results are listed in Table 1 as well. Obviously, both helium and in particular metallicity are now much higher as compared to the previous case ignoring diffusion in the calibration; the latter values are increased by $10-13 \%$ (similar values for the comparison codes). We are using the same solar initial abundances 
for the M67 isochrones. This is not completely consistent as M67 displays now a solar metallicity, but due to its slightly younger age diffusion should have started with a somewhat lower metallicity than the Sun. However, this effect amounts to a few parts in $10^{-4}$ in $Z$ only, such that we did not iterate further the initial composition.

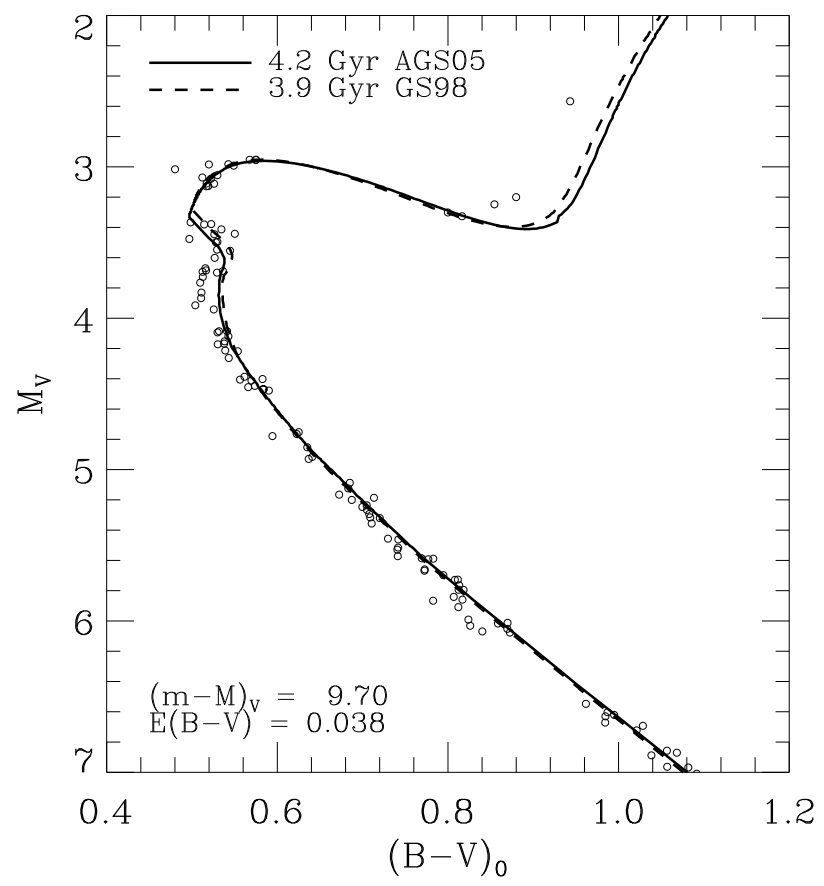

FIG. 2.- Isochrone fit to M67 using an initial composition obtained from standard solar model calibration with diffusion. Tracks for M67 include diffusion and NACRE rates; no overshooting was considered.

The final fits to M67 are shown in Figure 2. We still used the same distance and reddening values, but the isochrone ages are now lower by 0.3 Gyr compared to Figure 1. The agreement with the ages by VG07 is purely incidental. Instead, the age for the GS98 mixture of 3.9 Gyr should be compared to that by Michaud et al. (2004) of 3.7 Gyr. Now, for both compositions the CMD morphology is reproduced, and AGS05 is no longer disfavored. The fit itself is of the same quality as before.

\section{VARIATIONS}

In this section we investigate the importance of further physical and numerical aspects that influence the appearance and size of a convective core on the main sequence. These are obviously the nuclear reaction rates of the CNO-cycle and the amount of overshooting. Overshooting may lead to the persistence of the otherwise transient convective core that may appear towards the end of the pre-main sequence, but may be kept alive due to additional mixing of $\mathrm{CNO}$-nuclei by overshooting. With this aspect we start our tests.

\subsection{Overshooting}

We first repeated the models of $\S 3.1$, i.e. diffusion was completely ignored, but included overshooting according to our standard prescription outlined in $\S 2.1$

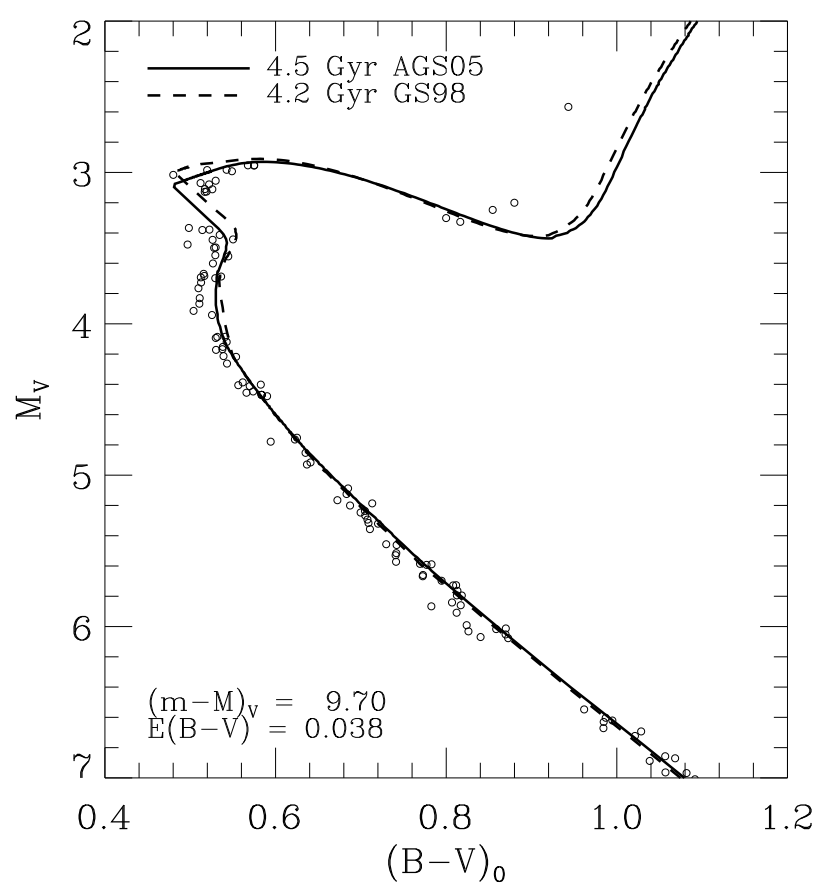

FIG. 3.- As Fig. 1 but here overshooting with a parameter of $f=0.018$ and geometric cutoff was used.

Equations 1 and 2, The parameter $f$ can be varied, but we keep it below the standard value of 0.02 (Herwig et al. 1997). In this work we take $f=0.018$ because with this value, in our alternative approach (Equation 3), we are able to reproduce well the evolutionary tracks from Pietrinferni et al. (2004), as mentioned in $\S$ 2.1. In Figure 3 the resulting CMD for $f=0.018$ and the standard cluster parameters is displayed. We recall that the amount of overshooting is reduced due to our geometrical restriction (equation 2); this reduction can be quite restrictive for stars with lower masses, around $1.2 M_{\odot}$, and be crucial in this study. However, even if the fit itself is not as good as before, now both mixture cases have TO masses with a convective core being present.

The situation seems to be typical for cases without diffusion in both the solar calibration and the cluster models. In VandenBerg \& Stetson (2004) the authors concluded that a small amount of overshooting is needed to reproduce best the CMD of M67. Overshooting was treated following the integral criterion by Roxburgh (1989) and the adaption of a varying amount of overshooting for very small convective cores close to $M_{\mathrm{ccc}}$, determined by VandenBerg et al. (2006). We stress the fact that this procedure was calibrated using open clusters with solar abundances on the old GS98 scale. For complete consistency, this procedure should be repeated for the new AGS05 composition, and this may explain why the same small amount of overshooting used by VG07 did not lead to a persistent convective core in case of the new abundances.

The case of $\S 3.2$, i.e. the one with full consideration of diffusion, was also extended by including overshooting in the tracks for M67. We refrain from showing the 


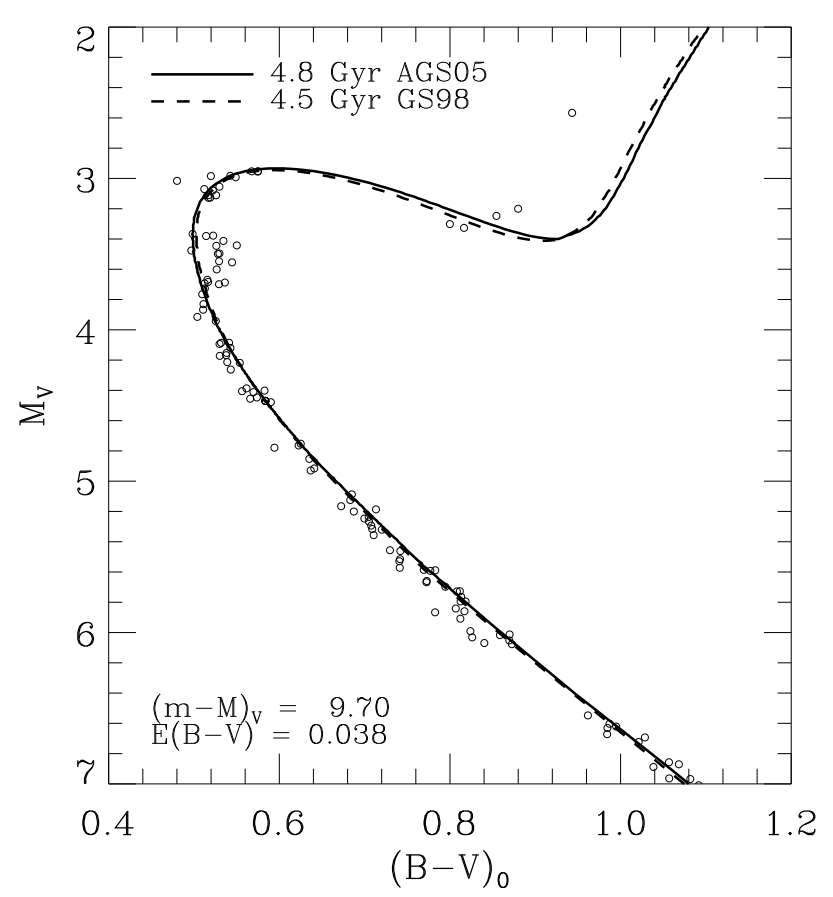

FIG. 4.- CMD of M67 with isochrones resulting from tracks computed with the ${ }^{14} \mathrm{~N}(p, \gamma){ }^{15} \mathrm{O}$ reaction rate by Marta et al. (2008) instead of that in the NACRE-library. In both the solar calibration and these tracks diffusion and overshooting were ignored.

result here, as for both mixtures the convective core, as expected, persisted, but the overall fit got worse. This coincides with the isochrone fit shown by Pietrinferni et al. (2004), where the CMD could be better reproduced if overshooting was completely ignored. It agrees also with Michaud et al. (2004), who neither found any necessity for overshooting, and who, too, did include diffusion in their models.

\subsection{Nuclear reaction rates}

As mentioned above, the nuclear reaction rates of the CNO-cycle are equally important for the occurrence of a convective core as is the abundance of CNO-nuclei. So far we have presented models employing the NACRElibrary reaction rates for these reactions. The bottleneck reaction of the CNO-cycle, ${ }^{14} \mathrm{~N}(p, \gamma){ }^{15} \mathrm{O}$, that determines the overall cycle rate, has been measured at stellar energies in the laboratory by the LUNA collaboration (Formicola et al. 2004; (Marta et al. 2008), and was found to be lower by about $50 \%$ with respect to the NACRE rate. The consequences for globular cluster age determinations and for some sensitive phases of low- and intermediate-mass star evolution has been investigated by Imbriani et al. (2004) and Weiss et al. (2005).

The rate being lower, we expect that the transition mass to harboring a convective core increases. Therefore isochrones using the lower CNO-abundances of the AGS05 mixtures will be even less likely to show the TO hook. We repeated the case of $\S 3.1$ (i.e. ignoring diffusion completely) with the updated and most likely more accurate rate by Marta et al. (2008). The resulting CMD for M67, using again our standard distance mod-

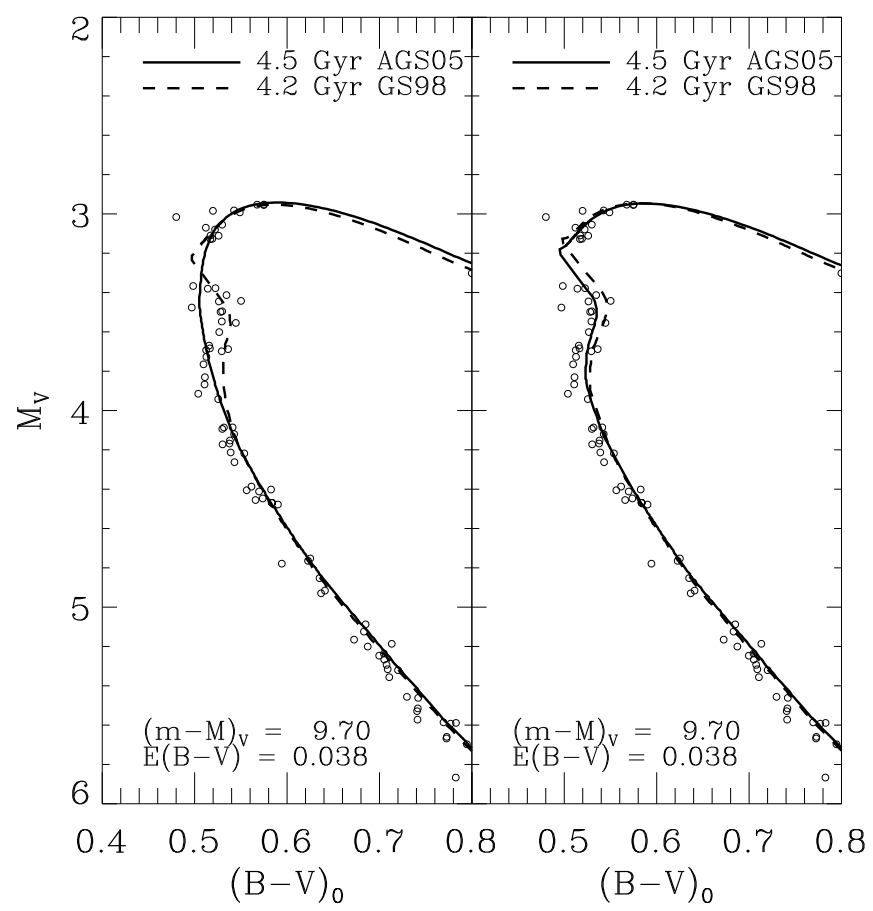

FIG. 5.- As Figure 4 but diffusion was included in both the solar calibration and in the tracks for the M67 isochrones. Left panel: overshooting constrained by the geometric cutoff (Equation 2); right panel: overshooting constrained by a linear dependence on stellar mass (Equation 3).

ulus and reddening is shown in Figure 4. In agreement with Imbriani et al. (2004) the isochrone age had to be increased (by 0.3 Gyr for both mixtures) and $M_{\text {ccc }}$ increased by $\approx 0.08 M_{\odot}$ in both cases. As a consequence also the GS98 case now lacks the characteristic hook. We add briefly that the inclusion of overshooting does not alter this result because the geometric cutoff is restrictive enough that the convective region formed at the end of the pre-main sequence phase can not be maintained during the main sequence evolution. The values for $M_{\mathrm{ccc}}$ are 1.215 (GS98) and 1.258 (AGS05), those at the TO 1.202 resp. $1.196 M_{\odot}$.

This illustrates the fundamental problem with such tests: Imagine we would assume that the GS98 solar composition is the correct one, but we want to test which reaction rate is to be preferred. From Figures. 1 and 4 we would clearly conclude that the older one is to be preferred!

We now repeat the computation of $\S 3.2$ i.e. the case with diffusion included, but with the newer and lower ${ }^{14} \mathrm{~N}(p, \gamma)^{15} \mathrm{O}$ reaction rate. In this case we recognize that with the older solar composition a small convective core is present $\left(\mathrm{TO}-\mathrm{mass} / M_{\mathrm{ccc}}=1.214 / 1.172\right)$, which is completely absent in the AGS05 case $\left(1.201 / 1.241 M_{\odot}\right)$. While the CMD fit is not very good even with the GS98 isochrone, it can be improved by including overshooting. The results, including overshooting with the geometric cut, are shown on the left panel of Figure 5 . As mentioned in the previous section, the geometric cutoff limit for overshooting is very restrictive particularly for lower masses. Therefore we recompute the tracks with the al- 


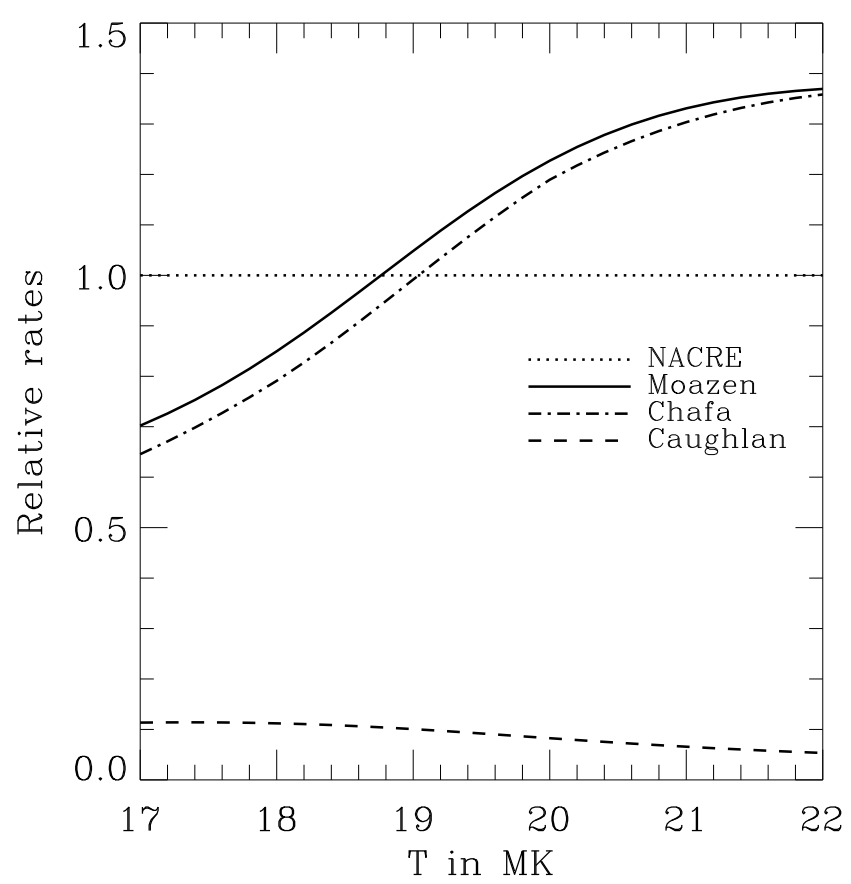

FIG. 6.- Rates for the nuclear reaction ${ }^{17} \mathrm{O}(p, \alpha){ }^{14} \mathrm{~N}$ relative to that of the NACRE library, in the interesting temperature regime for core hydrogen burning in typical M67 stars near the TO mass.

ternative limiting algorithm, the ramp function described by Equation 3. Changes in TO morphology for the GS98 composition are only minor. On the other hand, for the AGS05 composition, we now get a similarly good CMD fit as with the old mixture (right panel of Figure 5), as the convective core that appears in the pre-main sequence can be maintained during main sequence evolution.

We found a second reaction that is also influencing the efficiency of the CNO-double cycle, though indirectly. This is the ${ }^{17} \mathrm{O}(p, \alpha){ }^{14} \mathrm{~N}$ reaction that closes the CNO-II cycle and which is, together with the competing ${ }^{17} \mathrm{O}(p, \gamma){ }^{18} \mathrm{~F}$ the slowest one of that subcycle. For this reaction new cross section measurements by Moazen et al. (2007) and Chafa et al. (2007) are available. The resulting two new reaction rates are quite similar to each other and vary in the interesting temperature range of $15-22$ MK between $50 \%$ and $120 \%$ of the NACRE rate. Additionally, we have also considered for this reaction the rate given by Caughlan \& Fowler (1988, CF88). The comparison of all rates is shown in Figure 6.

In Figure 7 we show the evolutionary track of a $1.2 \mathrm{M}_{\odot}$ stellar model with GS98 composition for different choices of the two key nuclear reactions mentioned above. The track corresponding to our standard choice of nuclear rates, NACRE, is shown by the dotted line. Now, if the CF88 rate for the ${ }^{17} \mathrm{O}(p, \alpha)^{14} \mathrm{~N}$ reaction is used, which is less than $10 \%$ of the NACRE rate throughout the relevant temperature regime, the track for this crucial mass is strongly modified and no longer shows a sign of a convective core, as depicted by the long-dashed line. If the Moazen et al. (2007) rate is used, the differences with respect to the track using NACRE rates are very small; for this reason, we do not show this track. However, it

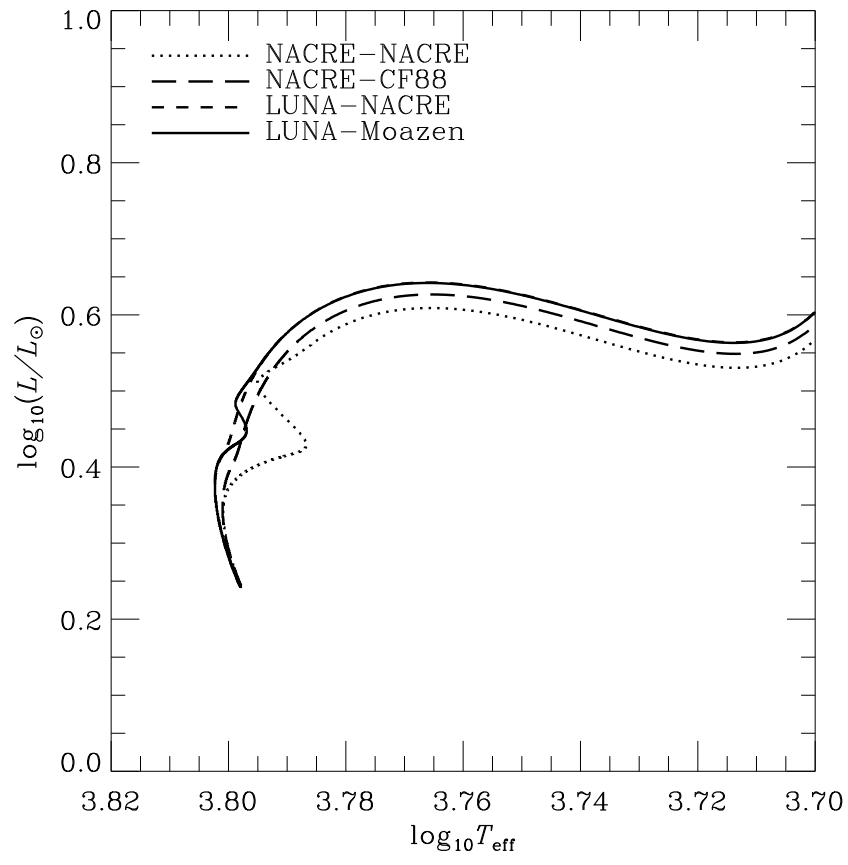

FIG. 7.- Evolution of a $1.2 M_{\odot}$ star with GS98 composition, neglecting diffusion and overshooting, and using different rates for the two bottleneck reactions of the CNO-cycle (see text for details). In the figure, the legends denote the source for the reaction rates: left refers to the ${ }^{14} \mathrm{~N}(p, \gamma){ }^{15} \mathrm{O}$ reaction, bottleneck of the whole CNO-cycle; right refers to the ${ }^{17} \mathrm{O}(p, \alpha){ }^{14} \mathrm{~N}$ reaction, bottleneck of the CNO-II cycle.

is interesting to note that the effect that changing the ${ }^{17} \mathrm{O}(p, \alpha){ }^{14} \mathrm{~N}$ rate has on the tracks depends on the rate used for the ${ }^{14} \mathrm{~N}(p, \gamma){ }^{15} \mathrm{O}$ rate. Indeed, as stated before, using the LUNA rate for this reaction (and NACRE rates for all others) leads to the absence of a convective core in the evolution of a $1.2 M_{\odot}$ stellar model. This is shown in the short-dashed line in Figure 7. However, when in addition the Moazen et al. (2007) rate is used, a small convective core is apparent in the track, as shown by the solid line in the same figure.

We have identified the reason for this to be the following: the low ${ }^{17} \mathrm{O}(p, \alpha)^{14} \mathrm{~N}$ rate from $\mathrm{CF} 88$ slows down the CNO-II subcycle, reducing thereby the flux of ${ }^{14} \mathrm{~N}$ back into the CNO-I cycle, which produces the overwhelming majority of the energy. While the loss of energy from CNO-II is rather unimportant, the drainage of available ${ }^{14} \mathrm{~N}$ by up to $90 \%$ from the CNO-I cycle is significant for its efficiency. One may consider this as a storage of nitrogen in the form of useless ${ }^{17} \mathrm{O}$. This makes the appearance of a convective core more difficult, and thus raises $M_{\text {ccc }}$. Note that for the higher, older, ${ }^{14} \mathrm{~N}(p, \gamma){ }^{15} \mathrm{O}$ rate from NACRE this effect is not important; it appears that in this case the branching is favoring the CNO-I cycle in any case, and the CNO-II cycle is always negligible.

Since the importance of the ${ }^{17} \mathrm{O}(p, \alpha){ }^{14} \mathrm{~N}$ was rather surprising, and to make sure that our $\mathrm{H}$-burning network included all necessary reactions, we did a test calculation with a variant of our code (Alves-Cruz 2009, private communication) that includes an extensive p-capture network up to silicon. In particular, the ${ }^{17} \mathrm{O}(p, \gamma){ }^{18} \mathrm{~F}$ reaction, which is the branching reaction to the next higher 


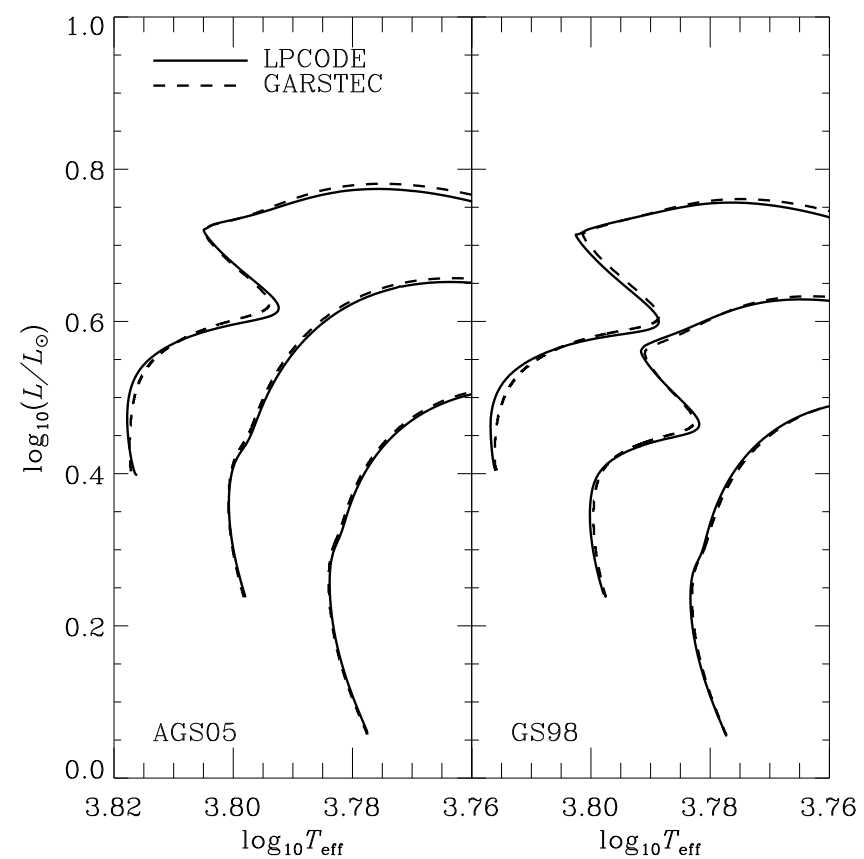

FIG. 8.- Comparison between LPCODE and GARSTEC. Here the tracks were computed with NACRE and the updated ${ }^{14} \mathrm{~N}(p, \gamma){ }^{15} \mathrm{O}$ reaction rate by Marta et al. (2008), diffusion, and overshooting, limited by the geometrical cutoff (Equation 2) and a parameter of $f=0.018$. Left panel: models with AGS05 composition; right panel: GS98 composition. In both cases tracks shown are $1.1,1.2$, and $1.3 \mathrm{M}_{\odot}$, which cover the critical masses for M67.

cycle, is included. We saw no difference in the evolution of stars in this mass range up to post main-sequence phase. Our treatment of the CNO-cycles thus appears to be complete and correct.

\subsection{Comparisons with other codes}

We have seen that the behavior of the convective core for masses around the TO mass in M67 is very sensitive to the constitutive physics included in the stellar models. Given this sensitivity, it is important also to determine if calculation done with different stellar evolution codes lead to the same conclusions. Here we have used, in addition to GARSTEC, results from two additional codes: LPCODE and the Dartmouth code. We summarize our findings below.

LPCODE has originally been developed at La Plata Observatory. Extensive descriptions of the code have been presented in Althaus et al. (2002, 2003). Here we only describe updates and changes done in the code that were specifically implemented for this work. The equation of state has been updated to the latest release by $\mathrm{OPAL}^{2}$. Radiative opacities from OPAL were calculated for both GS98 and AGS05 compositions and complemented at low temperatures with those from Ferguson et al. (2005). Conductive opacities, although not relevant for this work, are now those from Potekhin and collaborators, presented in Cassisi et al. (2007). For the nuclear reaction rates, NACRE is the standard choice but the LUNA rate for the ${ }^{14} \mathrm{~N}(\mathrm{p}, \gamma){ }^{15} \mathrm{O}$ from Marta et al. (2008) has been adopted when neces-

\footnotetext{
2 http://adg.llnl.gov/Research/ OPAL/EOS_2005/
}

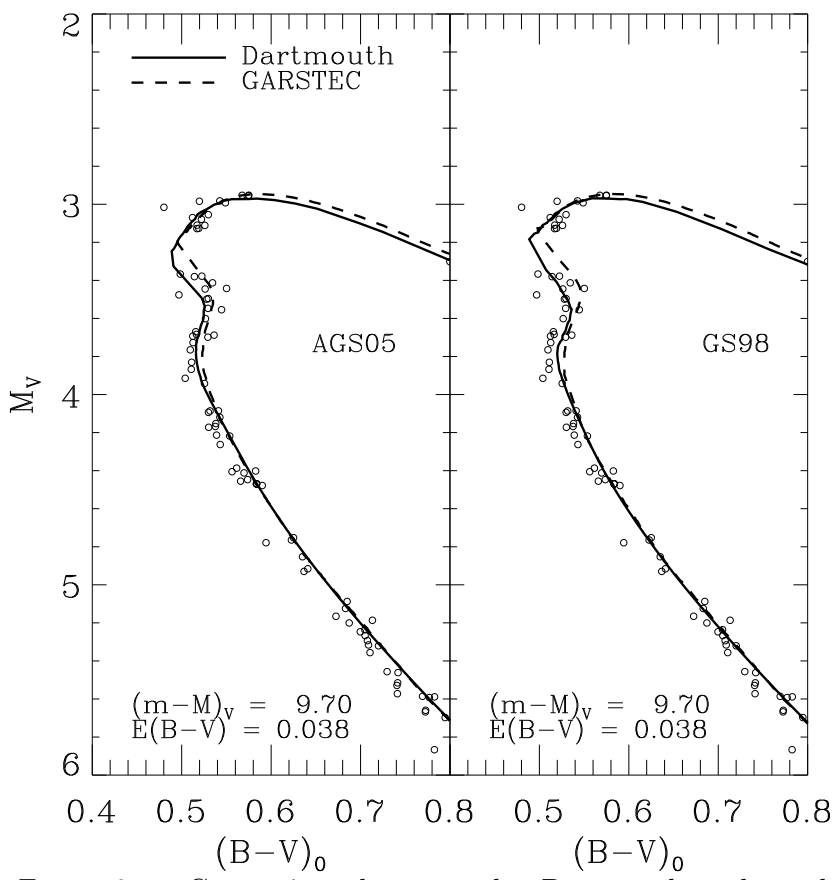

FIG. 9.- Comparison between the Dartmouth code and GARSTEC. CMDs of M67 showing, for AGS05 and GS98 compositions, isochrones from both stellar evolution codes including overshooting, diffusion and the LUNA rate for the ${ }^{14} \mathrm{~N}(p, \gamma)^{15} \mathrm{O}$ reaction (see text for more details). For each composition, morphology around the TO is similar for both codes. For AGS05, the derived age is 4.5 Gyr, while for GS98 is 4.2 Gyr (for both codes).

sary. Overshooting is treated in a diffusive approach but the geometric cutoff (Equation 2) has been implemented to allow better comparison with GARSTEC results.

We have computed a large set of models covering the ranges of mass, composition and input physics relevant to our work. In all cases, agreement between GARSTEC and LPCODE has been very good. Here, we choose to show in Figure 8 some evolutionary tracks that include the most complex input physics, as used for the CMD in in left panel of Figure 5 NACRE rates updated by the ${ }^{14} \mathrm{~N}(p, \gamma){ }^{15} \mathrm{O}$ reaction rate from Marta et al. (2008), diffusion in both the solar calibration and in the tracks, and overshooting limited by our geometrical restriction (Equation 2). Left and right panels show results for AGS05 and GS98 compositions, respectively. In both cases, tracks for $1.1,1.2$, and $1.3 \mathrm{M}_{\odot}$ are shown, that bracket the TO mass of M67 and, consequently, determine the morphology of the isochrones around the TO. By inspection of Figure 8 it can be readily seen that models with both codes show a very similar evolution along the HRD; differences are hardly noticeable. Results are of a similar quality for other choices of constitutive physics and for both composition options. In relation to systematic uncertainties affecting the conclusions of our work, this result is particularly encouraging because GARSTEC and LPCODE have been developed completely independently, and do not share any numerical algorithm for solving the equations of stellar evolution.

The Dartmouth stellar evolution code is derived from the Yale code (Guenther et al. 1992), with modifica- 
tions and updates described in Chabover et al. (2001), Biork \& Chabover (2006) and Dotter et al. (2008). For this project, the NACRE nuclear reaction rates were implemented, with the exception that the LUNA rate (Marta et al. 2008) for the ${ }^{14} \mathrm{~N}(p, \gamma){ }^{15} \mathrm{O}$ reaction was used. Convective core overshoot in the Dartmouth code is parametrized as a multiple of $H_{P}$. Normally, the prescription of Demarque et al. (2004) is used, in which the amount of core overshoot is small $\left(0.05 H_{P}\right)$ for stars with small convective core masses, and gradually increased to $0.20 H_{P}$ for stars with masses $0.2 \mathrm{M}_{\odot}$ above the critical mass for the turn-on of convection in the core. This prescription for convective core overshoot was found to yield good agreement with open cluster color-magnitude diagrams, when the Grevesse et al. (1996) solar mixture was used. This mixture has $(Z / X)_{\odot}=0.0244$. For this project, a small constant amount of convective core overshooting regardless of the stellar mass was used. The amount of convective core overshoot used in specific models is identified in the figure captions, and was typically $0.07 H_{P}$.

In the case of the comparison between results of GARSTEC and the Dartmouth code we choose to present CMD fits to M67 computed with both AGS05 and GS98 compositions. Models for GARSTEC use overshooting constrained by Equation 3 . Results for the best fit isochrones for both codes are shown in Figure 9, where the left (right) panel shows results for the AGS05 (GS98) composition. As throughout this paper, our goal is to determine whether isochrones based on stellar models with one or the other composition can reproduce the occurrence of the observed hook in M67. As can be seen in Figure 9. isochrones from both codes reproduce well the TO morphology for both compositions. In the case of AGS05, the age of for M67 obtained from both codes is $4.5 \mathrm{Gyr}$ and for GS98 is 4.2 Gyr. Although the TO morphologies are not identical for both codes, some differences are likely to be present since we have not attempted, even within the uncertainties given by the input physics and observational data, to obtain the best possible agreement between the codes and with the data.

As a last comparison, we state that the results determined in VG07 (see $§$ 3.1) could also be reproduced by both codes.

From comparing results from GARSTEC with those from LPCODE and Dartmouth code, we conclude that, even if numerical schemes for solving stellar struture and evolution equations and implementation of physics are different in the different codes, our conclusions are robust; they do not depend on the stellar code used. It implies that systematics between the codes are not an important source of uncertainty in our conclusions.

\section{DISCUSSION AND CONCLUSIONS}

The open cluster M67 has solar metallicity and its CMD shows a clear hook around the TO, evidence that stars populating the TO have convective cores. The mass of these stars is around $1.2 \mathrm{M}_{\odot}$, very close to the critical mass value for which a convective core developes as a result of the efficient operation of the CNO-cycle. Based on this, VG07 have suggested that M67 can be used to test the new revisions of solar abundances presented by Asplund and collaborators (Asplund et al. 2005, 2009), since $\mathrm{CNO}$ elements, catalysts in the CNO-cycle, have suffered the largest reductions in their solar abundance values. Here, we have reconsidered the viability of using M67 as a benchmark for solar abundances, by studying in detail under which conditions stars with masses around $1.2 \mathrm{M}_{\odot}$ develop (or not) a convective core towards the end of the main sequence and how this affects the morphology of isochrones best fitting M67 CMD.

We have first focused on reproducing the results obtained by VG07. Using initial compositions for both AGS05 and GS98 obtained from calibrating solar models without element diffusion, we have successfully recovered their basic results: if stellar models include a small amount of overshooting but no element diffusion, then the hook in M67 is not reproduced by isochrones computed with the AGS05 solar composition, while it is present if the GS98 composition is used since, in this case, stars populating the TO have a well developed convective core. This has been presented by VG07 as support for the GS98 solar composition; although VG07 pointed out to some caveats in their models that may influence this conclusion.

We then included element diffusion in our models. On the one hand, solar models calibrated with diffusion yield a higher solar initial metallicity, as summarized in Table 1. Additionally, because gravitational settling is the dominant effect, metals tend to accumulate in the core. Inclusion of diffusion has then a double effect towards compensating lower metallicities. Our models with diffusion, but still using NACRE nuclear rates, lead to the formation of a convective core for TO stars in M67 even with the AGS05 composition and, consequently, the distinctive hook observed in the CMD is also present in the isochrones, as shown in Figure 2. Under these assumptions, we find no clear evidence favoring the older, higher, GS98 solar metal abundances over AGS05.

In our study, we have also identified two nuclear reactions, ${ }^{14} \mathrm{~N}(p, \gamma){ }^{15} \mathrm{O}$ and ${ }^{17} \mathrm{O}(p, \alpha){ }^{14} \mathrm{~N}$, that are critical in determining the precise value of $M_{\mathrm{ccc}}$. The first reaction is the bottleneck of the whole CNO-cycle, while the second one is the bottleneck of the CNO-II part. When the temperature is not high enough, the latter acts as a sink for ${ }^{14} \mathrm{~N}$ nuclei, thereby slowing down the whole CNOcycle. Let us first discuss results related to the first reaction. In addition to the NACRE rate, we also used for ${ }^{14} \mathrm{~N}(p, \gamma){ }^{15} \mathrm{O}$ the latest measurement by the LUNA group (Marta et al. 2008), which is about a factor of 2 lower than the NACRE rate. As can be expected, the LUNA rate shifts $M_{\text {ccc }}$ to larger values, by about $0.08 \mathrm{M}_{\odot}$. With this rate, even models with GS98 composition (not including diffusion) lack the characteristic hook observed in M67 (Figure 44). This is not surprising in the light of results with AGS05 composition, since the effective operation of the CNO-cycle depends on the product of the abundance of catalysts and the ${ }^{14} \mathrm{~N}(p, \gamma){ }^{15} \mathrm{O}$ rate. The effect on the CNO-cycle of reducing the rate for this reaction by about a factor of 2 , for stars with masses close to $M_{\text {ccc }}$ is very similar as to reducing the total number of catalysts by a similar amount. A somewhat more unexpected result relates to the second reaction, ${ }^{17} \mathrm{O}(p, \alpha){ }^{14} \mathrm{~N}$. The branching between the CNO-I and CNO-II cycles is almost independent of temperature for the temperature range that interests us in this work. However, if the temperature is not high enough for the CNO-II cycle to be fully active, ${ }^{17} \mathrm{O}$ is created at the ex- 
pense of ${ }^{14} \mathrm{~N}$ but the feedback to the CNO-I cycle (the only one relevant in terms of energetics) is inefficient. As a consequence, the total number of catalysts is reduced, with ${ }^{14} \mathrm{~N}$ being stored in the form of ${ }^{17} \mathrm{O}$. For this rate we have used, in addition to the NACRE rate, the older rate by Caughlan \& Fowler (1988) and the new measurement by Moazen et al. (2007). When we use the Caughlan \& Fowler (1988) rate, that is about $10 \%$ of the NACRE rate in the relevant temperature range (see Figure 6 for a comparison of the rates), we find that, even for GS98 and the NACRE rate for ${ }^{14} \mathrm{~N}(p, \gamma)^{15} \mathrm{O}$ there is no convective core in a $1.2 \mathrm{M}_{\odot}$ models towards the end of the main sequence. This translates into the absence of a hook in the isochrones that would best fit M67 CMD. One can regard the last exercise as merely of academic interest since the low rate from Caughlan \& Fowler (1988) seems now to be ruled out by experiments. However, the importance of this rate is still worth being taken into account with the newest results by Moazen et al. (2007), even if this rate agrees with NACRE within 40\%. By using the Moazen et al. (2007) rate in the models, the $1.2 \mathrm{M}_{\odot}$ model with the LUNA rate for the ${ }^{14} \mathrm{~N}(p, \gamma){ }^{15} \mathrm{O}$ reaction recovers a small convective core. These results are summarized in Figure 7.

The development of convective cores in stars close to $M_{\text {ccc }}$ is sensitive to the detailed physical input in the models, as seen from the above results. Moreover, an appreciable amount of freedom in the modeling is still present because of the lack of a proper convection theory to account for, particularly, the amount of overshooting occurring in stellar cores. It is widely accepted, however, that an overshooting region of about $0.2 H_{P}$ is needed in stars with masses above $\sim 2 \mathrm{M}_{\odot}$ to explain, among others, the width of main sequences in stellar clusters. On the other hand, for stars below that mass, and particularly close to $M_{\mathrm{ccc}}$, it is also known that overshooting has to be limited to a smaller region. However, a detailed understanding of how core overshooting depends of stellar parameters, namely the stellar mass, is not known. We have used here two different approaches to limit overshooting, a geometric cutoff and an efficient factor that increases linearly with stellar mass, Equations 2 and 3 respectively, with the geometric cutoff being the standard choice. In relation to core overshooting, its importance is that it helps to reproduce the hook in M67 because it facilitates stars to sustain a convective core during the main sequence and the growth of the core towards the end of the main sequence for stars with masses around $M_{\text {ccc }}$, critical for explaining the TO morphology in M67. On the other hand, overshooting cannot, by itself, create a convective core when there is none. This is the case, for example, for our models with AGS05 composition and the LUNA rate for the ${ }^{14} \mathrm{~N}+p$ reaction when the geometric cutoff is applied, as shown in the left panel of Figure 5. In this case, the convective core developed towards the end of the pre-main sequence evolution cannot be sustained by stars with masses close to the TO mass in M67, even with the help of overshooting. This may be the result of our geometric cutoff being too restrictive. When, instead, the linear ramp function of Equation 3 is used, isochrones based on AGS05 show again the characteristic hook of M67 (right panel in Figure 5). With this prescription for overshooting, for stellar masses around M67 TO masses, the convective core survives during all main sequence evolution and naturally grows towards the end of it, due to increased CNO burning, leading to the occurrence of a hook in M67 CMD. Overshooting provides a certain degree of freedom in stellar models, and here we show that this freedom renders isochrone fitting to M67 uncertain, in that depending on the way overshooting is modeled, we are able to find, or not, a hook around the TO. It is important to note that both prescriptions for the limitation of overshooting for stellar masses around $M_{\text {ccc }}$ can be made consistent with current observational evidence.

We have also tried to assess the possible dependence of our results on the numerics, that is the stellar evolution code. Therefore, we have used, in addition to GARSTEC, two other evolution codes: LPCODE and the Dartmouth code. None of these codes share a common background in their development. For this work, when possible, we have tried to match the input physics, but some differences still remain. The most important, probably, is that in the Dartmouth code overshooting is accounted for as a fraction of the pressure scale height. For models appropriate for M67, this fraction has been taken as $0.07 H_{P}$. Comparison of results between the three codes is highly satisfactory. Solar model callibrations, summarized in Table 1, yield very small differences. A comparison between evolutionary tracks computed with LPCODE and GARSTEC is shown in Figure 8. The agreement between results from the two codes is excellent. A comparison of isochrone fitting to the CMD of M67 with GARSTEC and the Dartmouth code is shown in Figure 9 for both compositions used in this work. Again, results are similar with both codes, which are able to reproduce the observed morphology around the TO. For each composition, both codes also yield the same age for the cluster, 4.5 and 4.2 Gyr for AGS05 and GS98 compositions respectively. We conclude from these tests that systematics originating in the use of different numerical schemes, and even some differences in the implementation of the input physics, do not affect our conclusions.

Finally, we comment on two additional points that reinforce our main conclusions given below. As stated in $\S 2.2$, the uncertainty in the metallicity of M67, according to Gratton (2000) is about 0.06 dex, i.e. $15 \%$. We have not played with the metallicity of our models and have always assumed a solar value (where solar means GS98 or AGS05). However, in view of the dependence of the precise value of $M_{\mathrm{ccc}}$ to the details of the constitutive physics, variations in the metallicity of our models can in principle affect the occurrence or not of a convective core for stars with M67 TO-mass. The last point is that in the new determination of solar abundances by Asplund et al. (2009) CNO elements have been revised upwards. The total number of CNO catalysts in the new Asplund et al. (2009) is about 8\% higher than in the previous AGS05 solar abundance compilation. This increase would also contribute to facilitate the occurrence of a convective core at the TO-mass in M67.

The main conclusions we draw from our study are as follow. The different solar compositions, namely AGS05 and GS98 certainly have an impact on the predicted morphology of the CMD in M67. The occurrence of the TO-hook is more difficult to achieve for the low CNO abundances of AGS05. This is a confirmation of previ- 
ous results by VG07. However, we have also found that other constitutive physics in the models, e.g. element diffusion, nuclear reactions, prescription of core overshooting, also influence the stellar mass at which convective cores start to develop. This translates, in the case of M67, into isochrones that may have a hook around the TO even with the AGS05 composition or, on the contrary, that show no hook even for the GS98 composition. There is a certain degeneracy in the constitutive physics underlying the presence of the hook in M67 that cannot be, at the present status of our knowledge, disentangled. The morphology of M67 could in principle be used to test solar abundances, but only under the strong assumption that all other factors affecting the TO morphology, more precisely the occurrence of a convective core in stars with masses around the TO-mass, are completely under control. Since we understand this is not the case, we conclude that M67 CMD morphology does not present a strong argument against low $\mathrm{CNO}$ abundances in the Sun.

We wish to thank Don A. Vandenberg for his very helpful input to this work and his openess to discuss our results and compare them with his own. M. Alves-Cruz did additional calculations, which we gratefully acknowledge. We also thank M. Asplund, S. Cassisi and L. Pasquini for helpful discussions and advice.

\section{REFERENCES}

Alecian, E., Lebreton, Y., Goupil, M., Dupret, M., \& Catala, C. 2007, A\&A, 473, 181

Althaus, L. G., Serenelli, A. M., Córsico, A. H., \& Benvenuto, O. G. 2002, MNRAS, 330, 685

Althaus, L. G., Serenelli, A. M., Córsico, A. H., \& Montgomery, M. H. 2003, A\&A, 404, 593

Anders, E. \& Grevesse, N. 1989, Geochim. Cosmochim. Acta, 53, 197

Angulo, C., et al. 1999, Nuclear Physics A, 656, 3

Antia, H. M. \& Basu, S. 2006, ApJ, 644, 1292

Asplund, M., Grevesse, N., \& Sauval, A. J. 2005, in Astronomical Society of the Pacific Conference Series, Vol. 336, Cosmic Abundances as Records of Stellar Evolution and Nucleosynthesis, ed. T. G. Barnes, III \& F. N. Bash, 25-+

Asplund, M., Grevesse, N., Sauval, A. J., \& Scott, P. 2009, ARA\&A, 47, 481

Bahcall, J. N., Basu, S., Pinsonneault, M., \& Serenelli, A. M. 2005a, ApJ, 618, 1049

Bahcall, J. N., Basu, S., \& Serenelli, A. M. 2005b, ApJ, 631, 1281

Bahcall, J. N., Serenelli, A. M., \& Basu, S. 2005c, ApJ, 621, L85

Basu, S. \& Antia, H. M. 2004, ApJ, 606, L85

Bergbusch, P. A. \& Vandenberg, D. A. 1992, ApJS, 81, 163

Bjork, S. R. \& Chaboyer, B. 2006, ApJ, 641, 1102

Caffau, E., Ludwig, H., Steffen, M., Ayres, T. R., Bonifacio, P., Cayrel, R., Freytag, B., \& Plez, B. 2008, A\&A, 488, 1031

Cassisi, S., Potekhin, A. Y., Pietrinferni, A., Catelan, M., \& Salaris, M. 2007, ApJ, 661, 1094

Cassisi, S., Salaris, M., Castelli, F., \& Pietrinferni, A. 2004, ApJ, 616,498

Caughlan, G. R. \& Fowler, W. A. 1988, Atomic Data and Nuclear Data Tables, 40, 283

Centeno, R. \& Socas-Navarro, H. 2008, ApJ, 682, L61

Chaboyer, B., Fenton, W. H., Nelan, J. E., Patnaude, D. J., \& Simon, F. E. 2001, ApJ, 562, 521

Chafa, A., et al. 2007, Phys. Rev. C, 75, 035810

Chaplin, W. J., Serenelli, A. M., Basu, S., Elsworth, Y., New, R., \& Verner, G. A. 2007, ApJ, 670, 872

Christensen-Dalsgaard, J., di Mauro, M. P., Houdek, G., \& Pijpers, F. 2009, A\&A, 494, 205

Demarque, P., Woo, J., Kim, Y., \& Yi, S. K. 2004, ApJS, 155, 667

Dotter, A., Chaboyer, B., Jevremović, D., Kostov, V., Baron, E., \& Ferguson, J. W. 2008, ApJS, 178, 89

Ferguson, J. W., Alexander, D. R., Allard, F., Barman, T., Bodnarik, J. G., Hauschildt, P. H., Heffner-Wong, A., \& Tamanai, A. 2005, ApJ, 623, 585

Formicola, A., Imbriani, G., Costantini, H., Angulo, C., D.Bemmerer, Bonetti, R., Broggini, C., \& et al. 2004, Phys. Lett. B, 591, 61

Freytag, B., Ludwig, H., \& Steffen, M. 1996, A\&A, 313, 497

Gratton, R. 2000, in Astronomical Society of the Pacific Conference Series, Vol. 198, Stellar Clusters and Associations: Convection, Rotation, and Dynamos, ed. R. Pallavicini, G. Micela, \& S. Sciortino, 225-+

Grevesse, N. \& Noels, A. 1993, in Origin and Evolution of the Elements, ed. S. Kubono \& T. Kajino, 14-+
Grevesse, N., Noels, A., \& Sauval, A. J. 1996, in Astronomical Society of the Pacific Conference Series, Vol. 99, Cosmic Abundances, ed. S. S. Holt \& G. Sonneborn, 117-+

Grevesse, N. \& Sauval, A. J. 1998, Space Sci. Rev., 85, 161

Guenther, D. B., Demarque, P., Pinsonneault, M. H., \& Kim, Y. 1992, ApJ, 392, 328

Gustafsson, B. 2003, in IAU Symposium, Vol. 210, Modelling of Stellar Atmospheres, ed. N. Piskunov, W. W. Weiss, \& D. F. Gray, 3-+

Guzik, J. A. 2008, Memorie della Societa Astronomica Italiana, 79, 481

Herwig, F., Bloecker, T., Schoenberner, D., \& El Eid, M. 1997, A\&A, 324, L81

Iglesias, C. A. \& Rogers, F. J. 1996, ApJ, 464, 943

Imbriani, G., et al. 2004, A\&A, 420, 625

Kippenhahn, R. \& Weigert, A. 1990, Stellar Structure and Evolution, A\&A Library (Heidelberg: Springer)

Maiorca, E., Caffau, E., Bonifacio, P., Busso, M., Faraggiana, R., Steffen, M., Ludwig, H., \& Kamp, I. 2009, Publications of the Astronomical Society of Australia, 26, 345

Marta, M., et al. 2008, Phys. Rev. C, 78, 022802

Michaud, G., Richard, O., Richer, J., \& VandenBerg, D. A. 2004, ApJ, 606, 452

Moazen, B. H., et al. 2007, Phys. Rev. C, 75, 065801

Montgomery, K. A., Marschall, L. A., \& Janes, K. A. 1993, AJ, 106,181

Percival, S. M., Salaris, M., \& Kilkenny, D. 2003, A\&A, 400, 541

Pietrinferni, A., Cassisi, S., Salaris, M., \& Castelli, F. 2004, ApJ, 612,168

Randich, S., Sestito, P., Primas, F., Pallavicini, R., \& Pasquini, L. 2006, A\&A, 450, 557

Roxburgh, I. W. 1989, A\&A, 211, 361

Sandquist, E. L. 2004, MNRAS, 347, 101

Sarajedini, A., von Hippel, T., Kozhurina-Platais, V., \& Demarque, P. 1999, AJ, 118, 2894

Socas-Navarro, H. \& Norton, A. A. 2007, ApJ, 660, L153

Twarog, B. A., Anthony-Twarog, B. J., \& Edgington-Giordano, F. 2009, PASP, 121, 1312

VandenBerg, D. A., Bergbusch, P. A., \& Dowler, P. D. 2006, ApJS, 162,375

VandenBerg, D. A. \& Clem, J. L. 2003, AJ, 126, 778

VandenBerg, D. A., Edvardsson, B., Eriksson, K., \& Gustafsson, B. 2008, ApJ, 675,746

VandenBerg, D. A., Gustafsson, B., Edvardsson, B., Eriksson, K., \& Ferguson, J. 2007, ApJ, 666, L105

VandenBerg, D. A. \& Stetson, P. B. 2004, PASP, 116, 997

Ventura, P., Castellani, M., \& Straka, C. 2005, A\&A, 440, 623

Ventura, P., Zeppieri, A., Mazzitelli, I., \& D’Antona, F. 1998, A\&A, 334,953

Weiss, A. \& Schlattl, H. 2008, Ap\&SS, 316, 99

Weiss, A., Serenelli, A., Kitsikis, A., Schlattl, H., \& ChristensenDalsgaard, J. 2005, A\&A, 441, 1129

Young, P. R. 2005, A\&A, 444, L45 